\title{
Computational Models of Dementia and Neurological Problems
}

\section{Włodzisław Duch}

Department of Informatics, Nicolaus Copernicus University, Torun, Poland School of Computer Engineering, Nanyang Technological University, Singapore. Google: Duch

\begin{abstract}
The final goal of neuroscience is to fully understand neural processes, their relations to mental processes and to cognitive, affective, and behavioral disorders. Computational modeling, although still in its infancy, already plays a central role in this endeavor. A review of different aspects of computational models that help to explain many features of neuropsychological syndromes and psychiatric disease is presented. Recent advances in computational modeling of epilepsy, cortical reorganization after lesions, Parkinson's and Alzheimer diseases are reviewed. Some trends in computational models of brain functions are identified.
\end{abstract}

Key words: neural networks; cognitive computational neuroscience; associative memory models, cortical reorganization, computational models in psychiatry, Parkinson disease, Alzheimer disease.

\section{A bit of history}

Neuroinformatics has two large branches. On the one hand it provides tools for storage and analysis of information generated by neuroscience. On the other hand it provides simulations and models that capture some aspects of information processing in the brain. Complexity of the brain dynamics may be too high to understand brain's 
functions in details in conceptual terms. Computational models based on correct principles may capture progressively larger number of essential features of brain dynamics, eventually leading to models of the whole brain that no individual expert will ever be able to understand in details. This situation is analogous to the cell biology, where the sheer number of biomolecules and their interactions will prevent experts from understanding all genetic and metabolic mechanism of a living cell. From the engineering perspective understanding a complex system implies the ability to build a model that behaves in important aspects in the same way as the system that is being modeled. At this point in history computer simulations are the easiest way to build complex models, but progress in building neuromorphic devices that implement some neural functions in hardware may change this situation in future (1).

In 1986 two volumes "Parallel Distributed Processing: Explorations in the Microstructure of Cognition" (2), written mostly by psychologist, were published. The first volume of the PDP book (as it was commonly called) focused on general properties of parallel information processing, drawing analogies with human information processing. The Parallel Distributed Processing (PDP) name did not gain popularity, replaced by "connectionism", the name that stressed the importance of connections that pass information between network of nodes representing neural cell assemblies, increasing or inhibiting their activations. One of the chapters described now famous "backpropagation of errors" algorithm that can be used to train a network of simple artificial neurons with a one-way (feedforward) signal flow desired responses to incoming signals. Such artificial neural networks became very useful for medical diagnostics support, signal and image analysis and monitoring, search for carcinogenic agents and other data analysis tasks. In these applications neural networks face strong competition from statistical, pattern recognition, machine learning, and other mathematical techniques.

The second volume of the PDP book contained models of psychological processes (speech perception, reading, memory, word morphology and sentence processing) and biological mechanisms (neural plasticity, neocortex and hippocampus models). In this area neural models have little competition, with exception of continuum neural field theory (CNFT) that treats neural tissue as a substrate in which various excitations are propagated. Although this theory contributes to the understanding of global models of the cortex and it gives rise to interesting phenomena that may be experimentally observed (3) neural field theory is not yet sufficiently developed to account for properties of biological memory, not to mention more complex functions.

The two PDP volumes gave rise to a great interest in formal neural network models, connectionist modeling in psychology inspired by the parallel distributed competitive brain-style information processing, and computational neuroscience, that focus on the biologically plausible models of single neurons or small neural assemblies. These neural models are fairly detailed, up to the level of ionic channels and specific neurotransmitters, producing spikes that have very similar characteristics to those observed in vivo. The question "at which level should one model the brain" obviously depends on the purpose of such modeling, and thus cannot have general answer. It would be very interesting if various artificial neural networks based on graded (non-spiking) neurons could be derived as an approximation to biologically plausible models. A common assumption is that an average number of spikes per second represents neural activity, and therefore artificial neural network models may 
use neurons that are characterized by a single number reflecting their activity. Potentially important phase relations between spikes and differences between dynamics of various types of synapses and neurotransmitters are lost in this way. There are many other approximations to biological neural processes that remain to be explored.

Despite these difficulties simple models of neural networks are surprisingly successful providing insight into various brain functions. One year after the PDP book appeared Ralph Hoffmann published in the Archives of General Psychiatry the first paper that used neural network model to understand psychiatric problem, the schizophrenia-mania dichotomy (4). In 1988 National Institute of Mental Health (NIMH) initiated "Computational, Theoretical and Mathematical Neuroscience program", supporting computer simulations in psychiatry, and the first international workshop on neural modeling of cognitive and brain disorders, sponsored by the National Institutes of Health, was held at the University of Maryland in 1995, resulting in the first book on this topic (5). At the same time inspirations derived from recurrent neural networks treated as dynamical systems started to penetrate brain science $(6,7)$ and even developmental psychology $(8,9)$.

Computational psychiatry is thus quite young, and bearing in mind the differences between the style of thinking in psychiatry and computational modeling it may take a long time before it will become a part of the mainstream psychiatry. Only a small percentage of the authors of papers in books devoted to this topic are professional psychiatrist $(5,6,10)$. Nonetheless, almost all neuropsychological syndromes and psychiatric disorders have reasonable computational models generating useful hypotheses that can be verified by experimental work. Spiking neurons (11) and sophisticated biologically plausible models of neurons (12-14) applied to understanding of brain dysfunctions show the overlapping interest of computational neuroscience and psychiatry.

Before reviewing the current state of the art in computational psychiatry a short discussion in section two introduces key concepts and problems in this field. This is then followed by the discussion of work on neurological problems and Alzheimer disease, with more emphasis on the understanding and ideas generated with the help of computational models than on technical details. Future prospects of computational psychiatry are discussed in the final section.

\section{How to model brain functions}

All models of brain functions have to face the tradeoff between simplicity and biological faithfulness. Simplicity implies the ability to understand in conceptual terms how and why the model works the way it does, leading to new ideas that may grasp the imagination of researchers for a long time. Lateralization of brain functions (left-right hemisphere division) found its way into popular psychology books. McLean's theory of the triune brain (15) introduced in the 1950's division into archipallium, reptile-like brain stem functions, paleomammalian limbic system functions, and neocortex, the rational mammalian brain. Although the concepts of the limbic system is rather vague and is severely criticized (16) simplified theories are still prevalent. Computer models are usually constructed to account for results of a single experiment and have always a limited range of applicability. Computer simulations may rarely lead to wide-ranging theoretical concepts, but they may provide simpli- 
fied, metaphoric language that facilitates thinking about brain processes. Brain-as-acomputer information processing metaphor has been replaced by new metaphors: brain as a connectionist system, dynamical system, and self-organizing system (17). These metaphors penetrated now deeply into psychology (7-9) and make their ways slowly into psychiatry. They can be used to describe dynamics of neural cell assemblies, but also global activity of the brain, for example mood swings in bipolar disorders (18). Such simple models are quite far from biological realism but may still be quite useful providing metaphorical language that reflects neurodynamical processes.

Biological faithfulness requires large scale neural simulations at the sufficient level of details to guarantee comparison with experimental results obtained by neurophysiologists. Until recently such simulations were possible only for quite small networks composed of a few dozens of neurons, but in 2005 projects proposing simulation of whole cortical minicolumns with $10^{4}-10^{5}$ neurons and $10^{8}$ connections on teraflop supercomputers were formulated (for example, the IBM Blue Brain project (19)). Such detailed simulations, if successful, should allow to make experiments in silico that will be very difficult or even impossible to perform in vivo. It is therefore possible that answers to fundamental questions, such as the nature of the neural information codes, will be found using computational models. Once these questions will be solved simplified architectures for brain-like computing, as well as models for different disorders, could be constructed.

\section{$2.1 \quad$ Neural models}

Traditional neuropsychological theories describe information processing in the brain using box-and-arrow diagrams, treating brain dysfunctions as disturbances in the information flow. Biophysical models may be very detailed, aiming for biologically faithfulness in many aspects. Majority of neural and connectionist brain function models are somewhere in between. They are usually based on inspirations by, rather than systematic approximations to, biophysical models. Successful connectionist models capture principles behind biological realization of certain brain functions, providing proofs that postulated mechanisms may in principle explain some results of observations and experiments, without quantitatively predicting any variables that may be directly measured.

A network of nodes interacting with each other through connections of different strength can collectively do interesting computations. Some of these computations involve:

- transforming the incoming signals: filtering irrelevant information, aggregating information to construct new features, enhancing salient features;

- categorizing elementary signals (such as phonemes or graphemes) to simplify analysis of complex signals in discretized form;

- self-organizing to reflect general topographical properties of sensory inputs and motor actions;

- memorizing associations at many levels, providing models of different types of distributed, content addressable memory;

- making decisions, setting goals and controlling behavior. 
Each network node, frequently called "an artificial neuron", may represent either a single neuron of a specific type, a generic neuron, a group of a few neurons, a neural assembly or an elementary function realized in an unspecified way by some brain area. Two key features of neural models are internal signal processing done by their nodes, and the type of interactions among these nodes. Signal processing mechanisms that neurons (network nodes) internally possess are a form of internal knowledge representation allowing for transformation of the incoming signals. In the simplest case this knowledge is specified by just a single parameter, a threshold for activation of a neuron by the incoming signals. This threshold is an "adaptive parameter", which means that it is adjusted by the learning algorithm of the model to improve performance of the task the whole network is required to do. Real neurons send single spikes when the sum over all incoming signals in a given time window (called neuron's activation) exceeds certain threshold value. This fact, and the brain-computer analogy, gave rise to the logical neuron model, introduced by McCulloch and Pitts (for anthology of early papers on neurocomputing see (20)). If the activation of a logical neuron is below the threshold its output is zero, and if it is above the threshold its output is one. Networks of such logical neurons may implement arbitrary Boolean function, but training such networks is fairly difficult. Single spikes do not matter much, and the frequency of spikes is approximately proportional to the activation of the neuron around the threshold activation value, going to zero for activation value significantly below the threshold, and saturating at maximum frequency for high activation values. This gives rise to the graded response neuron models, with output defined by a non-linear tilted S-shaped (sigmoidal) function of activation. The response gain of the neuron is defined by the steepness of this function. Because the output functions are squashing linear activations to keep all outputs between zero and some maximum values they are sometimes called "squashing functions".

It is clear that such simplified models loose important features of biological networks, such as the ability to synchronize the activity of several units for specific time delays between spikes (phase relations). Models of real neurons at different abstraction level, from simple "integrate and fire" neurons without specific spatial structure, to multi-compartmental models that take into account geometry of dendrites and axons, are available in NEURON and GENESIS software packages (12-14). Sophisticated biophysical models of compartmental neurons provide information directly related to neurophysiological parameters measured in experiments. Already in 1994 Callaway and collaborators modeling reaction times to different drugs stated: "Neural network models offer a better chance of rescuing the study of human psychological responses to drugs than anything else currently available" (21). Such detailed models related to psychiatric problems are available only in rare cases (a few papers may be found in books $(5,6,10)$ and $(22))$.

Connectionist models go in quite different direction, as their nodes are not related to single neurons, representing rather memory states realized as a joint activity of neural ensembles. High activity of such nodes may represent recognition of letters, phonemes, words or iconic images. These models provide a bridge between neural and symbolic information processing, rule-based systems for systematic reasoning and grammatical analysis. Knowledge stored in nodes of connectionist networks is still rather simple, although many parameters may be required to represent it. In the 
limit each node may become an agent, with more internal knowledge and some methods to process this knowledge depending on the information received (23).

The second main aspect of neural models is the communication between the nodes. Signals carrying information about activity of input units may flow through the network in one direction, from designated inputs, through some hidden elements that process these signals, to output elements that determine the behavior or signify decisions of the network. In logical networks signals have only two values, True and False, and connections between two logical neurons may either leave them unchanged, or negate them. By analogy to biological neurons connections are sometimes called "synaptic", direct connections are "excitatory" and negated connections "inhibitory". Activity of the neuron is simply calculated as the number of true inputs received by the neuron minus the number of the false inputs. This activity is then compared with the threshold to determine whether the neuron should output the signal True or False. Although this is a very simple model threshold logic implemented by such neurons allows for definition of non-trivial concepts, such as "the majority" (at least half of the inputs should be True to output True value).

Graded neurons are used in the popular Multilayer Perceptron (MLP) neural networks that assume feedforward flow of information between layers of neurons. Such architecture (Fig. 1) simplifies training of the network parameters that include thresholds and the strength of connections $W_{i j}$ between pairs of neurons $n_{i}$ and $n_{j}$. The only information that is passed through such networks is the strength of the signals $X_{i}$, and the only knowledge that neurons hold are activation thresholds $\theta_{i}$. MLPs are examples of mapping networks that transform input signals into output signals and may learn, given sufficient examples of inputs and desired outputs, how to set the connections $W_{i j}$ and the thresholds $\theta_{i}$ (or the interactions between the nodes and the local knowledge in the nodes) to perform heteroassociation between inputs and outputs. Learning algorithm of such networks is driven by errors that are assumed to be propagated from the output to the input layer, although such a process is difficult to justify from biological point of view. MLP networks may thus signify the presence of certain categories of inputs, reduce the amount of information needed for making decisions, or provide new set of features for further processing. 


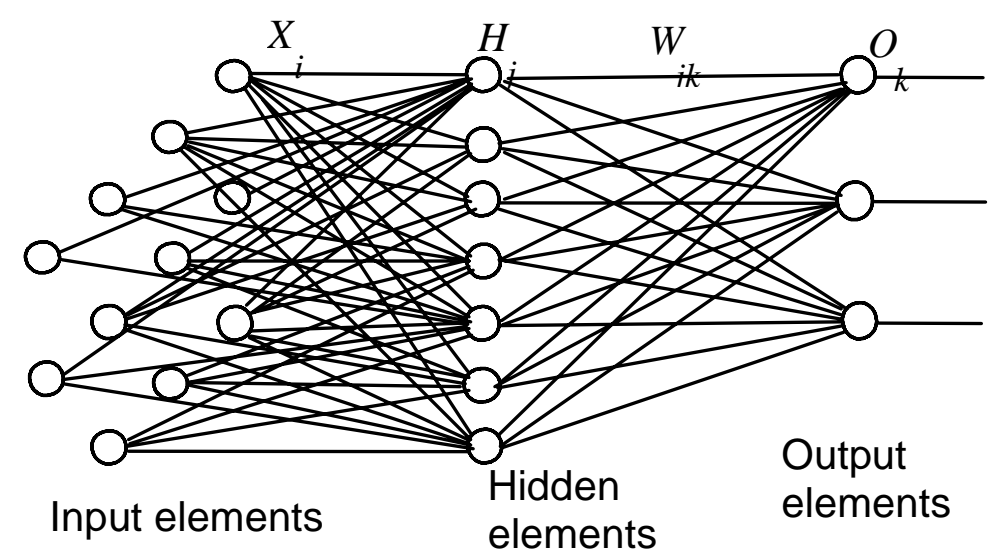

Fig. 1. Mulit-layer perceptron feedforward network with a single hidden layer.

Biological networks are almost never feedforward, containing many feedback loops, and assuming dynamical states that memorize or represent information received by the network. Given $n$ logical neurons in some initial state and connecting them all together one may observe how their mutual excitations and inhibitions evolve until either a static configuration of activities is reached and no further change is possible (point attractor), or cyclic changes occur indefinitely (cyclic attractor), or the system reaches chaotic state. In 1982 John Hopfield described such fully connected network of the two-state neurons (24). With additional assumptions that there are no self-connections and all weights are symmetrical the evolution of such network always ends in one of several static configurations, depending on the initial state and on the weights that connect neurons. Such networks are commonly called "the Hopfield networks", and are the simplest type of attractor neural networks, or dynamical systems motivated by neural ideas. A version of such network with graded neuron activities and continuous weights is very useful as a model of autoassociative memory. Learning in Hopfield type of network may be based on the Hebb principle that neurons that show correlated activity should have stronger excitatory connections, those that show negative correlation (one active when the other is not active) should have stronger inhibitory connections, and those that do not show any correlations may have weak connections or loose them completely. Therefore Hebbian learning is also known as the correlation-based learning. 


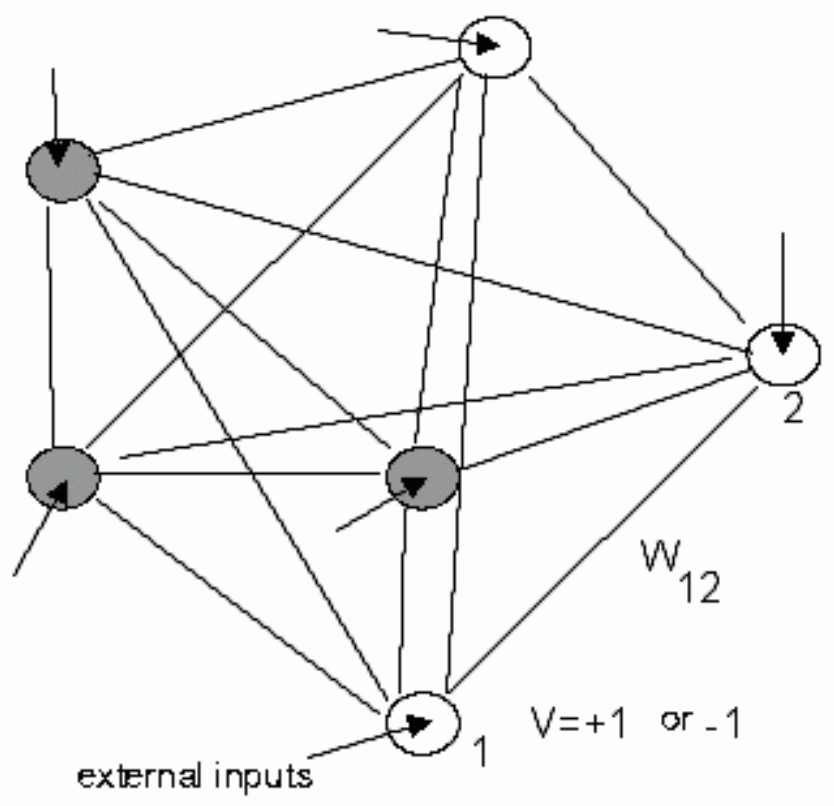

Fig. 2. Hopfield network with 6 neurons, 3 dark neurons are in an active state and 3 light neurons are in the inactive state.

The MLP and the Hopfield networks are frequently used in modeling of brain functions related to perception and memory. One more basic model that is very useful in understanding the development of topographical mappings has been introduced by Teuvo Kohonen (25), and is known as the Self-Organizing Mappings (SOM), or Kohonen network. It is also a very simple model, with two-dimensional grid of nodes (neurons) that do not communicate with each other directly (Fig. 3), but tend to react in a similar way when the training signals are presented. These neurons have no internal parameters, only weights $W_{i}$ that may be compared to the input signals. Evaluation of similarity, the basic operation in SOM network, may be viewed as a simplification of neural activation by a specific train of spikes. Initially all weights are random, but after presenting training signals to the network many times the learning mechanisms pulls together those weights that are similar to typical signals (centers of clusters in the input space). In effect different part of the neural grid show maximum activity for different types of signals, discovering for example the phonetic structure of speech signals. SOM networks may model spontaneous perceptual and motor learning in infancy, but may also be useful as a model of reorganization of cortical functions after stroke or loss of limbs (see below). 


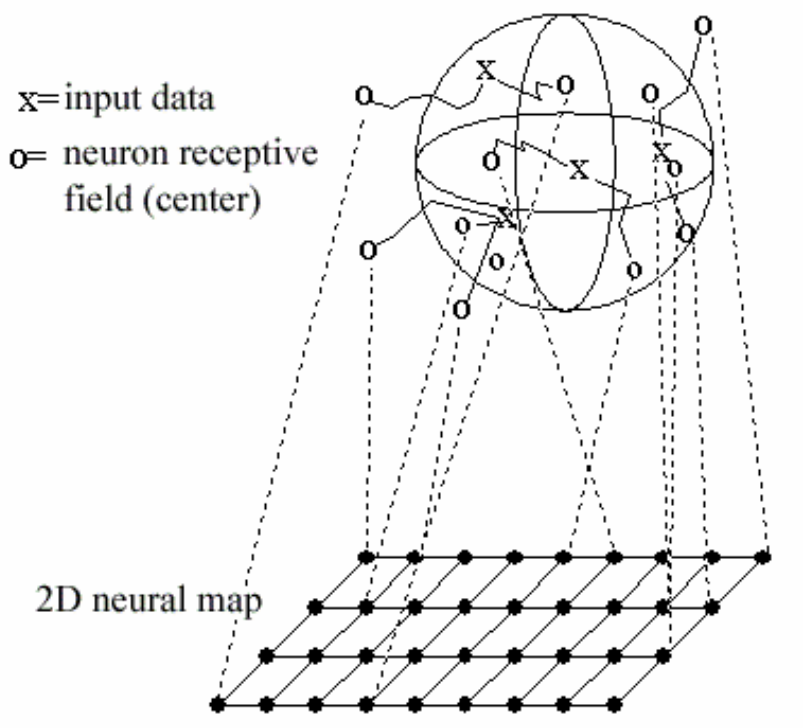

N-D feature space

Fig. 3. Self-Organized-Mapping is a grid of processors, each with parameters that learn to respond to signals from the high-dimensional input feature space.

These three type of models and their many variants are used extensively in the "Computational Explorations in Cognitive Neuroscience" book (26) that contains many experiments made with accompanying PDP++ software for biologically based modeling of psychological functions. Although it is not directed at psychiatric disorders it has been used to set up many experiments to elucidate various brain mechanisms involved in perception, attention, recognition, semantic, episodic and working memory, phonology and speech perception, reading and dyslexia, mapping phonological to orthographical representations, and executive frontal lobe functions.

\subsection{Brain simulations and mental functions}

Large neural networks based on biologically plausible spiking neural models may be used for modeling of many brain functions, but such models may not only be difficult to simulate, sometimes requiring supercomputing power, but may also be rather difficult to analyze and understand. Although simple approaches presented above are much less faithful to biology they may elucidate some brain mechanisms, generating useful hypothesis. Neural simulations should capture casual relations between activity of brain structures and their general neuroanatomical features, in particular influence of lesions and neuropathological changes on modification of normal behavior and cognitive performance. It is not a priori clear that simplified neural models will be sufficient to capture such casual relations. Convergence of the modeling process 
could be too slow to make them useful; for example some pathological effects could appear only in models based on complex multi-compartmental spiking neurons.

Fortunately there are some indications that the qualitative behavior of complex models based on spiking neurons (27) may also be obtained in simplified neural models (5). Even the simplest neural models of associative memory show many characteristics known from psychology, such as: content-addressability - cues lead to the whole memorized patterns, even when they are imperfect (computer memory needs an address to find information); graceful degradation - damage to some connections does not erase specific facts, only increases the recall error rates; time of recall does not depend on the number of memorized items (in computers time of recall is proportional to the number of items); similar items get more frequently mixed up; an attempt to understand too many things quickly leads to chaotic behavior, etc.

Thus there is a chance that simple neural models may help to understand neurological and neuropsychological syndromes, providing some insight into the source of pathologies and some understanding of the effects of therapeutic procedures. Classical methods of psychiatry and neuropsychopharmacology are restricted to observations of correlations between behavior and physiological responses of the organism to medical treatments. They usually try to understand the mechanisms leading to neuropathological behavior at the neural level, while cooperative network-level effects may be quite complex and difficult to infer from experiments. Brain simulations can complement traditional techniques in several ways. They provide insights into possible causal relations, allow for a full control of all aspects of experiments, they are inexpensive and are not restricted by ethical considerations. An early review article (28) and the books $(5,6,10,22,26,29)$ provide many insights into the mechanisms behind the memory and language impairments, psychiatric disorders, Alzheimer and Parkinson disease, epilepsy and other neurological problems.

Hierarchical approach to modeling of brain functions at different levels of complexity has been presented in (23) and (30), addressing the problem of creating understandable description of complex phenomena and the gap between neuroscience and psychology. Neural models may predict behavioral patterns but do not capture the inner, first-person subjective perspective, they do not offer any vision of mind. Transitions between quasi-stable states of attractor networks lead to behaviorist rules, reducing complex neurodynamics to simple symbolic description of animal and human behavior. Rules are very rough approximation to neurodynamics, but an intermediate level of modeling, presenting current state of the network in reduceddimensionality spaces, is possible. At the level of conscious decision making only some highly processed features are accessible, with active "mind objects" in psychological spaces reflecting neural dynamics. This line of modeling, still quite rare, is in line with Roger Shepard's search for universal laws of psychology in appropriate psychological spaces (31). It has been applied to analysis of psychological experiments on human categorization, providing both neurodynamical and psychological perspective of the processes responsible for puzzling human judgments (32). Although such mental-level models have a chance in future to complement neural models providing deeper understanding of brain dysfunctions so far they have not been used in this area.

In the remaining part of the paper a review of two applications areas of computational psychiatry is presented, various neurological problems and Alzheimer disease. 
This review illustrates the type of models that has been used, and emphasize insights, understanding and hypothesis generated by computational models.

\section{Neurological problems}

Neurological problems, such as epilepsy, ischemic or hemorrhagic strokes, cerebral palsy or parkinsonism, are usually associated with well defined anatomical and physiological changes in the brain. In this respect the modeling problem is easier than in case of such psychiatric disease as for example schizophrenia. An early review of different neurological problems has been presented in (33).

Perhaps most detailed models so far have been made for epilepsy, which is one of the most widespread neurological disorder, characterized by recurrent unprovoked seizures that frequently lead to the loss of consciousness. Many types of epilepsy exist, differing in region of origin of synchronous neural oscillations (usually hippocampus and certain neocortical regions), with frequency that may either be quite low, or extremely high (up to $600 \mathrm{~Hz}$ ). Dynamical neural networks with strong feedback connections and little inhibition may easily be brought to a threshold of epileptic-like discharge, but this is rather trivial observation. Much deeper and more detailed understanding is required. For example, it is known that small changes in the molecular structure of neuron's sodium ionic channels may be one of the causes of epilepsy, and some drugs act by modulating those currents. Therefore detailed models that include many pyramidal neurons with several types of ion channels are particularly useful here. Many such models have been developed in the Jeffreys $(34)$ and Traub $(35,36)$ groups. They are able to generate EEG patterns that closely resemble experimental ones, elucidating the role of various types of neurons and pharmacologic agents in epilepsy.

Generation of extremely high frequency oscillations is not possible in model networks without electrotonic synapses, much faster than the classical chemical synapses. Although it is known since the early 1980s that such synapses should exist (because blocking of chemical synaptic transmission does not stop the epileptic seizures), direct evidence for their existence in the neocortex is still controversial. Fast electrical nonsynaptic communication is possible through gap junctions filled with connexins, intramembranous proteins, that have rapidly modifiable conductance properties. Predictions of computational models are even more specific, gap junctions should exist between the axons of pyramidal cells in hippocampus and neocortex.

These hypothesis focus the search for therapeutic strategies on direct manipulation of gap junctions to decrease synchrony. A better understanding of molecular processes at the gap junctions may be achieved by detailed biophysical modeling techniques based on molecular dynamics.

Parkinson's disease is connected with the loss of dopaminergic neurons in the substantia nigra that project to one of the basal ganglia large nuclei, the putamen. As a result motor control problems develop, including akinesia (difficulty initiating movements), tremor, rigidity, slowing of movements and poor balance. In a substantial percentage of patients cognitive impairment, hallucinations and depression may occur. The origins of this disease are not yet clear and therapy is based on dopamine precursor, L-DOPA, that slows down the progress of the disease. 
Movements are smooth if the timing between activation of agonist and antagonist muscles is synchronized. Low level of dopamine in the putamen may lead to the imbalance in the cortico-basal-ganglia-thalamic-cortical loop that controls the motor system (involvement of the cerebellum in this loop is usually neglected). In computational models the dynamics of four layers of neurons was therefore investigated, with feedback from the fourth to the first layer (38). In such attractor networks change of parameters leads to sudden bifurcation, changing qualitative behavior of the network from point attractor to a cyclic attractor. Borrett et al. (38) interpret this change as the sudden onset of tremors when a significant decrease in dopamine level is reached. In their model it is preceded by a slower network response that may lead to slow movements. Edwards et al. (39) investigated richly connected inhibitory neural networks of the Hopfield type showing that such transitions between irregular and periodic dynamics are common if synaptic connections are weakened, because the number of units that effectively drives the dynamics is reduced, leading to simpler behavior. Fixed points in the dynamics of networks correspond to akinesia, while irregular dynamics may correspond to the normal low-level physiological tremor. Parkinson's high-amplitude tremor is regular and results from cyclic attractors in networks with simplified dynamics. Such models may produce variety of symptoms, relating them to different forms of cooperative behavior (see also papers in $(5,6)$ ).

Cognitive aspects of Parkinson's disease, and the influence of pharmacological therapy on cognitive abilities, have been modeled by Frank (40). Complexity of brain systems involved is to high to have a good verbal model of interactions between basal ganglia, motor cortex and prefrontal cortex. Although dopaminergic drugs ease the motor symptoms they may increase problems with execution of cognitive tasks. Basal ganglia is not only involved in motor actions, but also working memory updates and initiation of thought movement. This is a subtle system that normally operates with wide dynamic range of dopamine levels, but in Parkinson's patients taking dopaminergic medication this range is reduced, leading to frontal-like and implicit learning impairments. This model, implemented in the Leabra framework (26), provides many detailed testable predictions for neuropsychological and pharmacological studies.

Stroke and brain lesions have the longest history of computational modeling. The situation in case of focal cortical lesions in some areas is rather clear and detailed experimental data exist from animal and human studies. For example, in the somatosensory cortex topographical organization reflecting spatial relationships has been studied at many levels (see the review (41)). Cortical representations may reorganize as a result of lack of stimulation due to the nerve damage, limb amputation, direct lesion or other processes. The simplest model that leads to formation of topographical maps is based on the self-organized mapping networks (25) and has been used with success to model details of visual system development (42). SOM has been used in a number of studies involving cortical reorganization after lesions and stroke $(43,44)$.

More complex networks designed for cortical map reorganization with excitatory and inhibitory cells were used to model inputs from the hand, including fingers $(45,46)$. The model showed a number of physiologically correct responses, such as expansion and contraction of representations of the stimulated areas, effects of nerve deafferentation and gradual disappearance of the "silent" regions in the map, as seen in experiments. Self-organizing models with competitive activation and representa- 
tion of thalamus (a relay of sensory inputs) account also for the "inverse magnification" rule: the size of cortical representation is inversely related to the size of the cortical cells receptive fields, leading to large cortical representations devoted to the skin areas with small receptive fields (43). In this model thalamic and cortical areas are represented by two-dimensional sheets of neurons, folded into a toroidal shape to avoid the border areas. Each of the network nodes should stand for a microcolumn of about 110 neurons, with hexagonal 32x32 node network. Thalamic neurons, activated by external inputs from the skin, excite cortical receptive field composed of a central neuron and 60 neurons surrounding it in 4 rings. These thalamo-cortical connections are trained using competitive learning, while cortico-cortical connections have local excitatory connections to the nearest neighbors, and inhibitory connections to the further neighbors (the shape of the function describing the type of connections resembles a Mexican hat, with broad peak and a dip before leveling off). Effects of lesions may be investigated either by cutting the thalamic connections or by removing all connections of a group of neurons, which simulates their death. The remaining neurons surrounding the lesioned region do not receive the inhibitory inputs, therefore their dynamics changes quickly and they become more responsive to weak stimulations from the thalamic area. As a result of these activations slower synaptic learning processes lead to reorganization of the cortex responses, with neurons close to the lesioned areas partially taking over the function of the dead neurons. This reorganization may be faster if only those skin areas that lost their representation in the somatosensory cortex are repeatedly activated. Simple devices that provide tactile, vibration, and temperature stimuli placed over selected parts of the skin should speed up rehabilitation and prevent formation of phantom limbs (see below).

Reorganization due to the diffuse branching thalamic projections is limited to no more than $1 \mathrm{~mm}$, but experimental evidence shows that after a long time reorganization processes may extend to over $10 \mathrm{~mm}$. Cortical areas devoted to hand that has been amputated may for example start to specialize in face. It is not yet clear how exactly the reorganization process happens, but it is quite likely that new connections due to sprouting (and may be even due to the development of new neurons) are formed. Of course it is quite easy to put such effects into computational models, but without experimental constraints models can provide explanations that have nothing to do with reality. On the other hand some models are over-constraint by their assumptions, for example neglect of inhibition between cortical neurons (45).

Intriguing effects have been experimentally observed as a result of anesthesia: receptive fields are immediately expanding to neighboring neurons even before additional stimulation. This is probably due to the presence of weak connections between thalamus and topographically adjacent cortical areas and the lack of inhibition from neurons that become silent. Similar effect are also observed in visual cortex when artificial scotoma is created - the fill-in processes makes it invisible after several minutes (33). In this case the lack of inhibition, making neurons more responsive, leads to changes in the gain response of neurons.

A biologically plausible state-of-the-art model of the $3 \mathrm{~b}$ area of primary somatosensory cortex and thalamic nuclei has been constructed by Mazza et al. (47) using the GENESIS simulator. In this model cortex is composed of excitatory (regular and burst spiking) and inhibitory (fast spiking) neurons. All parameters of the model were taken from literature, therefore some aspects of simulations may be compared with in 
vivo measurements. NMDA, AMPA and GABA types of synapses were used and the synaptic changes observed during reorganization of maps after lesions. The hand mechanoreceptors give a short burst of action potentials when stimulated, adapting quickly to the pressure. A palm and four fingers containing together 512 receptors was simulated. The ventral posterior lateral (VPL) part of the thalamus contains excitatory neurons and inhibitory interneurons. The thalamic relay cell model contains a soma and three dendrites, with sodium, low threshold inactivating calcium, fast calcium, voltage dependent potassium, slow calcium dependent potassium and delayed rectifier potassium ionic channels. The thalamic interneuron has a soma and a dendrite with three type of ionic channels.

Cortical neurons are arranged in 3 layers (corresponding to the layer III, IV and $\mathrm{V}$ of the area $3 \mathrm{~b}$ ), with each layer composed of a $32 \times 32$ node grid of excitatory and inhibitory neurons. Three types of neurons were included: excitatory pyramidal cells, fast spiking inhibitory basket cell GABAergic neurons, and burst spiking excitatory stellate neurons, in total 3072 excitatory neurons and 1536 inhibitory neurons. Although real cortex contains more types of neurons these were selected as the most common.

Equations describing currents flowing through ionic channels have many parameters, but they are fixed using experimental values. Connectivity between these neurons is matched to the statistical information about real connectivity. Simulation of one second of real time processes took less than 4 hours on a personal computer. Many properties of normal maps are exhibited by this model, including stable representation of hand that has been developed in each cortical layer and in the thalamus nuclei. The most precise topographical maps occur in layer IV, and this is also shown in the model. Simulation of amputation of a finger showed immediate reorganization of the cortical (but not that much thalamic) representations, leading to expansion of the representation areas of the intact hand regions. This is followed by a slower improvement and consolidation of the new map. When a finger is removed the lack of activity from this finger removes inhibition, and the expansion follows. This process may be analyzed in all details, including the dynamics of the AMPA, NMDA and GABA receptors in the dendrites of model neurons. It appears that the information in the NMDA channel increases rapidly after lesion. This may be an important clue for pharmacological treatment of patients during rehabilitation.

Although the model is quite detailed it does not include such important features as long-term-potentiation (LTP). Of course the complexity of real neurons is orders of magnitude greater. Nevertheless, it is clear that models at this and higher levels of details will be more common in future.

Phantom limbs are experienced by some patients after amputation of arm, hand or breast. This curious phenomenon has been studied by Ramachandran (48). In the somatosensory cortex representation of hand is adjacent to the face. Patients that experienced phantom limb had also rather detailed representation of the hand in several places on their face, for example the sensation of touching a given finger could be elicited by touching three different well localized spots on the face. In another case sensation of touching the removed breast was elicited by touching the ear (49).

These sensations appears within hours after amputation and evidently shows that larger scale reorganization and rapid expansion of the cortical receptive fields took place rapidly. Some attempts at modeling this process have been based on self- 
organizing networks (50) with the reorganization processes driven by noise generated by dorsal root ganglion sensory neurons that fire irregularly after injury. Unfortunately this model has not yet been tested. It seems that more experimental data is needed to create detailed models of this phenomenon and gain more understanding of large-scale reorganization processes. Many questions arise, for example why only some patients experience it (due to stimulation of the skin?), or how to slow down the rapid reorganization (blocking NMDA receptors? avoiding stimulation o certain skin areas through local anesthesia?) to prevent appearance of the phantom limbs that create pain and discomfort to many patients.

Phantom limbs phenomenon is similar to another strange phenomenon, experience of additional ghost arm parallel to the normal arm. Despite apparent similarity the mechanism in this case seems to be quite different. The unitary percept of the body after stroke or other lesions may dissociate, creating an experience of additional arm that occupies the previous position of the real arm with a time lag of 60-90 seconds. fMRI study of one such stroke patient (51) with a right frontomesial lesion showed strong supplementary motor area (right medial wall) activity. This could be interpreted by the brain as an intention for movement of a hand that does not exist in this position.

Computational models of visual hemineglect and similar phenomena (52-55) may help to explain such experiences. Neglect patients, usually after lesions to the right parietal cortex, are not able to see objects in their left visual field if there is an object in their right field. In the particularly curious version of neglect, called objectbased visual neglect, patients do not see the left half of each object spread out horizontally in the visual field. Because this is object-based visual impairment attention mechanism have to be involved. Deco and Rolls (55) created a model of attention that included three areas: primary visual cortex (V1), object recognition areas (inferior temporal cortex, IT) and spatial orientation areas (posterior parietal cortex, PP). The spatially local lateral inhibition in the parietal and visual cortex produces at the edges of each object high contrast effects. If the damage in the parietal cortex increases linearly through the left visual field, local peaks in the resulting neuronal activation appear only for the right half of each object. There are many new models of attention (for example $(52,56)$ ) and thanks to the cooperation of experimental observations and computational modeling a better understanding of attention and executive function is slowly growing.

\section{Alzheimer disease}

Alzheimer disease (AD) is the most common neurodegenerative disorder gradually leading to a global cognitive and behavioral dysfunction. The disease is always progressive, without remissions but with great variability: life expectancy ranges between 1 and 25 years. The earliest symptoms involve memory degradation, both for learning new things and recalling known facts. This is followed by degradation of language skills, poverty of thoughts and associations, intellectual rigidity, loss of initiative and interest, disturbances in motor and executive functions. In advanced stages judgments are impaired, psychotic features may appear (such as paranoid delusions), and personality is disintegrated. 
The disease usually attacks first the entorhinal cortex and the adjacent limbic areas, and then spreads out to the neocortex. Prominent atrophy of predominantly frontal and temporal cortex is observed in neuroimaging studies and large amounts of senile plaques and neurofibrilliary tangles are found in the brain. Exact relations between cognitive decline and changes in the brain are rather complex and still not understood. Although knowledge of possible AD causes accumulates real causes of pathogenesis are still unknown. There are no reliable clinical tests and definitive diagnosis is made only after autopsy. Mild cognitive impairment, involving loss of short-term memory, represents an early stage of Alzheimer disease (57). Few drugs available specifically for Alzheimer treatment (for example Cognex and Aricept) do not slow the progress of disease directly, but are rather aimed at improving and stabilizing memory and cognitive state of the patient, helping to retain and utilize in a better way acetylcholine, one of the most important neurotransmitters (cholinergic neurons in the basal forebrain are destroyed in the early stages of $\mathrm{AD}$, resulting in low level of acetylocholine). Better understanding of the mechanisms and development of $\mathrm{AD}$ is thus necessary to propose better diagnostic and therapeutic procedures. This is a challenge to the neural modeling community because too little is known about the progression of the disease.

The neurofibrillary tangles and senile plaques are clear markers of the Alzheimer disease. Neuronal death is probably preceded by loss of synaptic connections. Earliest changes in the brain have been observed around the enthorinal cortex and the hippocampal formation. The first symptom of the mild cognitive impairment and of the AD itself concerns memory. All these facts point to the importance of the memory models $(58,59)$ in understanding Alzheimer's disease. Hippocampus is involved in memory consolidation and seems to be an intermediate memory storage that allows for formation of stable long-term memories without catastrophic forgetting of contradictory information (58). Neural models should unravel the relation between changes at the cellular level and various clinical manifestations of the disease. Three models of pathogenesis of AD have been proposed, all focusing on synaptic processes and their role in memory maintenance.

The "synaptic deletion and compensation" model of Horn et al. (60), developed further by Ruppin and Reggia (61), has been motivated by the following experimental observations. In the brains of AD patients the density of synaptic connections per unit of cortical volume decreases with progress of the disease, while the surviving synapses increase physically in size and thus provide stronger connections. It is likely that these synapses are trying to compensate for synaptic deletion. In feedforward neural network models pruning of weak synaptic connections is frequently used to improve their predictive powers. Pruning allows to forget the accidental details of mapping learned from data, while the essential characteristics are captured with simplified network structure using large synaptic weights of the remaining connections, necessary for realization of strongly non-linear behavior. How do these two processes - synaptic deletion and compensation - influence memory deterioration? What are the best compensation strategies that may slow down the memory deterioration process?

The simplest associative memory models that may be used to investigate such questions are based on the Hopfield networks (Fig. 2). Assuming that the synaptic matrix $W_{i j}$ determines the strength of connections between neurons $i$ and $j$, each of the $N$ neurons has threshold $\Theta_{i}$ for firing, and is in one of the two states $V_{i}= \pm 1$, the ex- 
ternal inputs are $E_{i}$, the total activation of neuron $n_{i}$ at the next time moment $t+1$ is a sum of all weighted activations at time $t$ of neurons that connect to it, minus the threshold for activation and plus the external input:

$$
I_{i}(t+1)=\sum_{j=1}^{N} W_{i j} V_{j}(t)-\Theta_{i}+E_{i}
$$

The simplest network dynamics is defined by taking the sign of the activation:

$$
V_{i}(t+1)=\operatorname{sgn}\left(I_{i}(t+1)\right)
$$

This dynamics has only point-attractor corresponding to the minima of the energy function:

$$
E(V)=-\frac{1}{2} \sum_{i \neq j}^{N} W_{i j} V_{i} V_{j}
$$

These stationary states are totally determined by the synaptic weights and thresholds, and may be interpreted as the memory patterns of the system: starting with activation pattern $V\left(t_{0}\right)=V_{0}$ that carries partial information about memorized pattern the system will evolve, changing neural activity in a series of steps until at some step the activity $V(t)$ does not change any more, reaching one of the memorized states.

The number of patterns ( $V_{i}$ vectors in the stationary states) that may be correctly memorized in the fully connected Hopfield autoassociative memory model is about $0.14 N$. Trying to memorize more patterns leads to chaotic, random associations. Deleting a large number of synaptic connections will cause forgetting of some patterns and distortion of others. Assume that a certain percentage $d$ of synaptic connections is randomly deleted (in the model their value is fixed at 0). Suppose also that some biological mechanism makes the remaining connections stronger. One way to express it is $W^{\prime}{ }_{i j}=c(d, k) W_{i j}$, where the compensating factor $c(d, k)>1$ is a multiplicative factor depending on $d$ and a parameter $k(d)$, called a compensation-strategy parameter, that is fitted to experimental data. Horn et al. (62) proved that taking $c(d, k)=k(d) d /(1-d)$ significantly slows the memory deterioration. Depending on the compensation strategy $k(d)$ after the same evolution period various degrees of deterioration are obtained. Thus failure of proper compensation for synaptic deletion may explain why patients with similar density of synaptic connections per unit of cortical volume show quite different cognitive impairments. This approach gives the modeler the freedom to fit $k(d)$ function to experimental data, but because the data is missing it simply shows what is possible, rather than what really happens in nature.

There are several problems with such simple model. Hopfield neural networks are not plausible from the neurobiological point of view because they require symmetric weights, have only point attractors and are trained using non-local learning procedures. Compensation quite efficiently maintains memory capacity even when more than half of the connections and neurons are deleted, while in the latest stages of AD no more than $10 \%$ of neurons are dead, but the density of synaptic connections may drop below $50 \%$ of the normal level. Addressing some of these questions Ruppin and Reggia (61), and Horn et al. (62) improved the compensation model in several ways, obtaining similar conclusions from other memory models (Willshaw, Hebbian, and modified Hopfield networks), with over 1000 neurons used in simulations. Activity-dependent Hebbian models allow for studying memory acquisition, showing such 
effects as faster forgetting of more recent memories. The memory recency effect, known in the psychological literature on memory as the 'Ribbot gradient', has been noticed a long time ago in retrograde amnesia (63-65), and has also been observed in Alzheimer's patients. Temporal gradients of memory decline and several other experimental phenomena characterizing memory degradation in AD patients have been recreated in Hebbian models. In such models there is no global error function that is optimized, local compensatory mechanisms are sufficient to maintain high capacity of memory (62). The way deletion and compensation factors change in time has an influence on the final performance of the network. Cognitive impairments are therefore history-dependent in this model, leading to a broad variability of the AD symptoms despite similar levels of structural damage of the brain.

Synaptic runaway model developed by Hasselmo $(66,67)$ is focused on a different phenomenon observed in associative memory attractor networks. To store a new pattern in the memory such networks explore first all similar patterns to find out if this is indeed a new pattern. If certain memory capacity is exceeded the new pattern may interfere with existing ones, creating an exponentially large number of slightly different patterns that the system tries to store. This initiates pathological, exponential growth of synaptic connections, known as the "synaptic runaway" effect. It is not clear whether such an effect exists in biological neural networks, but if it does, it should lead to a very high metabolic demands of hyperactive neurons, demands that in the longer time period cannot be satisfied. As a result toxic products should accumulate and neurons will die because of excitotoxicity creating senile plaques.

Synaptic runaway may arise due to excessive memory overload, reduced synaptic decay or a low level of cortical inhibition. If external synaptic strength is sufficiently large, or if internal inhibition is sufficiently strong, synaptic runaway may be prevented, but after critical storage capacity is exceeded it is unavoidable. This model explains some intriguing experimental facts about $\mathrm{AD}$ :

- Enthorinal regions (involved in recognition memory) suffer greater degradation than cortical areas and are usually impaired at an earlier stage; these regions lack internal inhibition present in cortical modules.

- Cholinergic innervation in dentate gyrus, the primary afferent area to the hippocampus, is sprouting in $\mathrm{AD}$ patients.

Acetylcholine (ACh) is a neurotransmitter that has complex functions. In dentate gyrus it does not influence external afferent synaptic transmission but it selectively suppresses the internal excitatory transmission, effectively increasing internal inhibition. Experiments that proved this were inspired by theoretical considerations of Hasselmo (67). Thus sprouting of cholinergic innervation may reflect the brain's attempts to stop the synaptic runaway by increasing internal inhibition. Another way to avoid this effect could be through separation of learning and recall mechanisms in the hippocampal networks. Perhaps acetylocholine level can switch networks between the two modes (68), with high levels present during active waking facilitating encoding new information in the hippocampus without interference from previously stored information (by partially suppressing excitatory feedback connections), and lower levels of ACh during slow-wave sleep facilitating consolidation of memory traces. 
Acetylocholine neuromodulation in the CA3 region of the hippocampus is also the focus of the Menschik and Finkel model (69-72). The neuroregulatory network is quite intricate and severely perturbed in Alzheimer's disease, involving death of neurons in several nuclei (locus coeruleus, dorsal raphe) that control norepinephrine and serotonin levels. ACh is produced (73) in medial septal nuclei and the vertical nucleus (diagonal band of Broca). Understanding of pathological effects arising in neuroregulatory networks requires detailed, biophysical models of neurons. Menschik and Finkel used parallel version o GENESIS to construct quite detailed model of hippocampal pyramidal neuron with 385 compartments with ionic channels of several types. Although this model was too complex for network computations some conclusions may be drawn even from single neuron simulations. For example, switching between learning and recall, corresponding to switching between burst and regular spiking, requires high level of acetylocholine that may not be available in hippocampus of the AD patient. Moreover, complete lack of ACh may lead to excitotoxic levels of calcium in dendrites.

Networks up to 1032 cells were constructed using 51 or 66-compartment neurons, based on the anatomy of the CA3 region of the hippocampus. The network was pre-wired in a Hopfield-like way to store some memory patterns, assuming that it has already undergone learning. This network behaves as an attractor network, with patterns presented as a series of spikes at the beginning of theta rhythm cycle, progressing to one of the stored cycles that appears as gamma bursts $(100 \mathrm{~Hz})$ within the theta cycle. In a large network 40 randomly chosen patterns of 512 bit each were stored. This network helps to assess the neuromodulatory role of acetylocholine on different ionic channels. Decline in Ach slows down the intrinsic gamma rhythm, and this in turn makes memory retrieval more difficult, giving less time for the network to settle in the attractor. Unfortunately after the initial burst of activity this interesting model has not been developed further.

All three neural models complement rather than compete with each other. Simple models that can be analyzed in details may be source of inspirations for more precise questions that biophysical models may answer. There may be several routes to development of Alzheimer Disease: synaptic loss and insufficient compensation should lead to AD cases with little structural damage of the brain, while synaptic runaway should eventually lead to death of the hyperactive neurons and significant structural damage. Both type of AD cases are indeed known and the great variability in the life expectancy and manifestations of clinical symptoms is probably a reflection of different underlying mechanisms.

Assuming that computational models reflect real neural mechanisms leads to several therapeutic suggestions, summarized in (74) and extended below. They may help to slow down the degeneration of synaptic connections and thus the development of the disease, at least in its early stages. These suggestions may be tested experimentally, and in view of high variability of the AD symptoms should be matched to individual cases.

If synaptic runaway processes and failure of proper compensation are the cause of rapid memory impairment then one should minimize new memory load for AD patients. This should involve regular, simple daily routine, and minimization of the number of new facts or items that should be remembered. Heavy memory load may contribute to the rapid progress of synaptic deletion. Patients should not be allowed to 
follow visual, auditory or printed stories, such as the TV news, soap operas or TV series that require remembering of many new facts, names and interpersonal relations. Sedatives may have positive effect on the memory overload because in the absence of strong emotions the limbic neuromodulatory systems does not increase synaptic plasticity, preventing formation of new memories. On the other hand activation of nondeclarative memory, for example by learning new skills, may work in a positive way. Engagement in new activity seems to benefit patients with mild dementia. Modeling this type of activity has not yet been attempted and it is likely that patients with different types of $\mathrm{AD}$ will respond in different ways

- $\quad$ Strengthen the old, well-established memory patterns.

A significant portion of time should be spent on recalling the stories and facts of patient's life with the help of family members. These memories form a skeleton of the concept of 'self'. Antonio Damasio (75) expressed it this way: “... the endless reactivation of updated images about our identity (a combination of the memories past and planned future) constitutes a sizable part of the state of self as I understand it". These memories are probably based on strong synaptic connections between cortical columns, with little involvement from limbic inputs required by more recent memories (cf. Murre $(63,76))$. Strengthening old memory patterns related to one's self is very much in line with the "Self-Maintenance-Therapy" (Selbst-Erhaltungs-Therapie) proposed by Romero (77) on quite different theoretical grounds and used in treatment of the early stages of Alzheimer's disease. In this therapy patients are required to tell stories recalling various events of their life as means to strengthen their self.

Compensation effects should selectively reinforce strong synaptic connections. This may be achieved through a combination of Self-Maintenance-Therapy (including family members) with drugs that allow for a short period of emotional arousal increasing synaptic plasticity.

- "Cool the brain": simplify the brain dynamics to avoid memory interferences.

Formation of new memory patterns or activation of existing memories requires repetitive high-frequency reverberations in the neocortex. For example, hearing and recognizing a real word leads to a noticeable rise in the EEG frequency, in comparison to a pseudoword, i.e. a meaningless combination of phonemes (78). Research on rats showed large effects of temperature on hippocampal field potentials (79). Integrated electrical activity of cortical columns gives a measure of the overall activity of the brain. The power spectrum obtained from the multi-electrode EEG measurements should allow, in the limit of a large number of electrodes, to evaluate this energy. In analogy to thermodynamics of systems far from thermal equlibrium one could thus define the "brain temperature" and think about the synaptic runaway processes as overheating the system. Direct measurements of the brain temperature may also show interesting differences between patients. Only recently a non-invasive technique for monitoring temperature via "Brain Temperature Tunnel" has been developed (80). Monitoring blood flow to the brain is an alternative way to estimate the total energy used by the brain. 
Blood pressure lowering drugs significantly decrease the risk of dementia, including Alzheimer's disease. This may be related to the decrease of overall brain temperature. Therefore 'cooling the brain', or reducing the average brain temperature, should decrease the effects of synaptic runaway and slow down the synaptic deletion processes. It may be achieved with the help of biofeedback, yoga meditation or other deep relaxation techniques. In particular the alpha-biofeedback is aimed at reducing the average EEG frequency (81), or achieving the 'alpha relaxation state'. Such mental activities as mantra repetition, chanting, visualization or contemplative absorption should lower the brain temperature, stopping the background thoughts and other processes that may lead to the synaptic runaway. There is a lot of evidence in medical literature showing various health benefits of such activities, and neuroimaging studies detailing the effect of relaxation response and meditative practices (82). Therefore in the early stages of $\mathrm{AD}$ it may be worthwhile to experiment with various relaxation techniques to slow down the development of the disease.

It should be possible to draw more detailed therapeutic suggestions from better models related to Alzheimer's disease. The existing models should be extended in several directions. Human memory involves interactions between hippocampal formation, neocortex and neuromodulatory systems, regulating plasticity of synapses depending on the emotional contents of the situation $(63,76,83)$. Such models have been initially created only at the conceptual level, but computational simulations followed $(64,65)$. More realistic memory models that would allow studying the influence of different neurotransmitters on the inter-module inhibition and betweenmodule excitation should help to evaluate potential benefits of new drugs. Models based on simplified spiking neurons are needed to make direct connections with neurophysiology. Many associative memory models based on simplified spiking neurons have been created recently and could be used in a near future to study the Alzheimer's disease and other memory-related diseases. Other approaches to AD are discussed in (84).

\section{Conclusions}

Neuroinformatics covers not only databases and computer software to analyze data from neuroscience experiments, but its ultimate goal should be an integration of all information about the brain (a good example here is the Visome platform (85)). This should include data from neuroscience experiments and tools for analysis of such data, but also tools for and results of computer simulations that provide models for various brain functions and dysfunctions. Simple neural models based on small number of assumptions allow for qualitative understanding of experimental observations in neuroscience and may help to generate interesting ideas for new experiments. Biophysical models capture sufficient amount of details to answer in silico precise questions that may be very difficult to answer experimentally.

A few models selected for this review show the potential of different approaches to neurological and psychiatric disorders, including Parkinson's and Alzheimer disease. An interesting area of neural modeling concerns reorganization processes following focal damages of neocortex (stroke, lesions) and damages to afferent pathways (amputation of limbs). Some therapeutic suggestions may be offered for faster recovery of sensorimotor competence after stroke (43), reduction of pain in phantom 
limb phenomena (44) and even such strange neuropsychological syndromes as the object-based unilateral hemineglect (55). Although therapeutic suggestions drawn here from AD models are speculative they are also worth testing.

Computer simulations appeared only quite recently as tool for modeling real brain processes. In view of the great complexity of the brain and lack of detailed understanding of its functions skepticism towards such models may seem to be justified. There are many fundamental problems related to the convergence of computational models, hypothesis on which they are based, selection of minimal neural models that capture relevant phenomena and are still amenable to computer simulations. Surprisingly, even very simple neural models of associative memory show a number of features that reflect many properties of real biological memories known from cognitive psychology. Therefore even rough neural models may show interesting properties, elucidating some brain mechanisms.

Neural models provide a new level of reasoning about brain diseases, level that cannot be adequately described in the language of psychiatry or psychopharmacology (30). They show how difficult it is to draw conclusions about causal mechanisms if only behavior is observed. Due to the limited capacity of human working memory verbal models, dominating in neuroscience, have to be relatively simple, and cannot incorporate too many factors and interactions. Computational models do not have such limitations and it is possible that such models will eventually capture all neuroscience knowledge, becoming repositories of collective effort of many experts (85). However, so far most computer simulations are aimed at explanation of a single experiment, or a single type of phenomena (with notable exceptions (86)), and they are frequently based on in-house computer algorithms and programs. Creation of flexible simulators that provide in silico models for a wide range of phenomena is a great challenge for the near future. There are already general purpose low-level modeling systems, such as NEURON or GENESIS, that provide models of specific dendrites, axons, whole neurons or small networks, but in most cases each new model has to be laboriously constructed from low-level elements.

There are two intermediate-level software packages designed for construction of computational models of brain functions. The PDP++ approach of O'Reilly and Munakata (26) has been used mostly to model different aspects of normal brain functions, but was also used recently for cognitive deficits in Parkinson's disease (40). The Neural Simulation Language (NSL), developed by Weitzenfeld, Arbib, and Alexander, provides another simulation environment for modular brain modeling, including the anatomy of macroscopic brain structures (87). It offers object-oriented language applicable to all levels of neural simulation, providing high level programming abstraction that use the Abstract Schema Language to create hierarchies of modules from the leaky integrator and other types of neural elements. Development tools are provided for visualization and analysis of models.

Availability of these type of neural simulators will eventually lead to development of a community of experts who will use them as primary tools for analysis and understanding of experimental data, as well as generating new ideas about normal and pathological brain functions. On the hardware side integrated circuits suitable for "neurophysiological" experimentations have already been constructed (88) and the whole neuromorphic sensory systems may slowly be developed (1). Computational 
models are certainly going to play an important role at every step of the long way leading to full understanding of the brain.

\section{Acknowledgements.}

I am grateful to the Polish Committee of Scientific Research for a grant (20052007) that partially supported this research.

\section{References}

1. Rasche, C. (2005) The Making of a Neuromorphic Visual System. Springer.

2. McClelland J. L. and Rumelhart D. E, eds. (1986) Parallel distributed processing: Explorations in the microstructure of cognition, Vol. I, II. MIT Press, Cambridge, MA.

3. Taylor J.G. (2003) Bubbles in the brain? Trends in Cognitive Neuroscience 7, 429-430.

4. Hoffmann, R.E. (1987) Computer simulations of neural information processing and the schizophrenia-mania dichotomy. Arch. Gen. Psychiatry 44, 178-188.

5. Reggia J.A, Ruppin E. and Berndt R.S, eds. (1996) Neural Modeling of Brain and Cognitive Disorders. World Scientific, Singapore.

6. Reggia J.A, Ruppin E. and Glanzman D.L., Eds. (1999) Disorders of Brain, Behavior, and Cognition: The Neurocomputational Perspective. Elsevier, New York.

7. Scott Kelso J.A. (1995) Dynamic Patterns. The Self-Organization of Brain and Behavior. Complex Adaptive Systems series. MIT Press, Bradford Book, Cambridge, MA.

8. Thelen E. and Smith L.B. (1994) A Dynamic Systems Approach to the Development of Cognition and Action. MIT Press, Cambridge, MA.

9. Smith L.B. and Thelen E., eds. (1994) A Dynamic Systems Approach to the Development. MIT Press, Cambridge, MA.

10. Parks R.W, Levine D.S. and Long D., eds. (1998) Fundamentals of Neural Network Modeling. MIT Press, Cambridge, MA.

11. Maass W, Bishop C. (1999) Pulsed Neural Networks. MIT Press, Bradford Book, Cambridge, MA.

12. Carnevale, N.T. and Hines, M.L. (2006) The NEURON Book. Cambridge University Press, Cambridge, UK.

13. Hines, M. (1993) NEURON - A program for simulation of nerve equations, in F. Eeckman (ed.), Neural Systems: Analysis and Modeling, Kluwer Academic Publishers, Norwell, MA, pp. 127-136. 
14. Bower J.M. and Beeman D. (1998) The Book of GENESIS: Exploring Realistic Neural Models with the GEneral NEural SImulation System. $2^{\text {nd }}$ ed, SpringerVerlag, New York.

15. MacLean P. (1990) The triune brain in evolution. Plenum Press.

16. LeDoux J. (1999) The emotional brain. Phoenix, London.

17. Erdi P. (2000) On the 'Dynamic Brain' Metaphor. Brain and Mind 1, 119-145.

18. Ehlers C.L. (1995), Chaos and complexity: Can it help us to understand mood and behavior? Arch Gen Psych. 52, 960-964.

19. IBM Blue Brain Project, http://bluebrainproject.epfl.ch/

20. Anderson J.A. and Rosenfeld E. (1988) Neurocomputing. The MIT Press, Cambridge, MA.

21. Callaway E, Halliday R, Naylor H, Yano L, Herzig K. (1994) Drugs and human information processing. Neuropsychopharmacology 10, 9-19.

22. Stein D.J., Ed. (1997) Neural Networks and Psychopathology, Cambridge University Press, Cambridge, U.K.

23. Duch W. and Mandziuk J. (2004) Quo Vadis Computational Intelligence? In: Machine Intelligence. Quo Vadis? Eds: P. Sinčák, J. Vaščák, K. Hirota. Advances in Fuzzy Systems - Applications and Theory - Vol. 21, World Scientific, 2004, pp. 3-28.

24. Hopfield, J.J. (1982) Neural networks and physical systems with emergent collective computational abilities. PNAS 79: 2554-2558.

25. Kohonen, T. (1995) Self-Organizing Maps. Springer Verlag.

26. O'Reilly R.C and Munakata Y. (2000) Computational Explorations in Cognitive Neuroscience: Understanding the Mind by Simulating the Brain. MIT Press, Cambridge, MA.

27. Xing J. and Gerstein G.L. (1996) Networks with lateral connectivity. I. Dynamic properties mediated by the balance of intrinsic excitation and inhibition. II. Development of neuronal grouping and corresponding receptive field changes. III. Plasticity and reorganisation of somatosensory cortex. J. Neurophysiology 75: 184-232.

28. Ruppin E. (1995) Neural Modeling of Psychiatric Disorders. Network 6: 635656.

29. Levine D.S. (2000) Introduction to Neural and Cognitive Modeling. $2^{\text {nd }}$ ed., Lawrence Erlbaum Associates.

30. Duch W. (1997) Platonic model of mind as an approximation to neurodynamics. In: Brain-like computing and intelligent information systems. Eds. S-i. Amari, N. Kasabov (Springer, Singapore), chap. 20, pp. 491-512. 
31. Shepard, R.N. (1987) Toward a Universal Law of Generalization for Psychological Science. Science 237, 1317-1323.

32. Duch, W (1996) Categorization, Prototype Theory and Neural Dynamics. Proc. of the 4th International Conference on SoftComputing'96, Iizuka, Japan, ed. T. Yamakawa, G. Matsumoto, pp. 482-485.

33. Crystal H. and Finkel L. (1996) Computational Approaches to Neurological Disease, In (5), pp. 251-272.

34. Jefferys, J.G.R. (1998) Mechanisms and experimental models of seizure generation. Current Opinions in Neurology 11, 123-127.

35. Traub R.D, Whittington M.A, Stanford I.M, Jefferys J.G.R. (1996) A mechanism for generation of long-range synchronous fast oscillations in the cortex. Nature 382, 621-624

36. Traub, R.D., Whittington, M.A, Buhl, E.H., LeBeau, F.E., Bibbig, A., Boyd, S., Cross, H. and Baldeweg, T. (2001) A Possible Role for Gap Junctions in Generation of Very Fast EEG Oscillations Preceding the Onset of, and Perhaps Initiating, Seizures. Epilepsia 42,153-170.

37. Bragin, A., Mody, I., Wilson, C.L. and Engel, J Jr. (2002) Local Generation of Fast Ripples in Epileptic Brain. J. Neuroscience 22, 2012-2021.

38. Borrett, D.S., Yeap, T.H. and Kwan, H.C. (1993) Neural networks and Parkinson's Disease. Canadian J. Neurol. Science 20, 107-113

39. Edwards R., Beuter, A. and Glass, L. (1999) Parkinsonian tremor and simplification in network dynamics. Bulletin of Mathematical Biology 61, 157-177.

40. Frank, M.J. (2005) Dynamic dopamine modulation in the basal ganglia: A neurocomputational account of cognitive deficits in medicated and non-medicated Parkinsonism. Journal of Cognitive Neuroscience 17, 51-72.

41. Buonomano D.V. and Merzenich M.M. (1998) Cortical plasticity: from synapses to maps. Annual Review of Neuroscience 21,149-86.

42. Erwin E., Obermayer K., Schulten K., (1995): Models of orientation and ocular dominance columns in the visual cortex: a critical comparison. Neural Computation 7, 425-468.

43. Reggia J, Goodall S, Chen Y, Ruppin E, Whitney C. (1996) Modeling PostStroke Cortical Map Reorganization. In (5), pp. 283-302.

44. Spitzer M. (1996) Phantom Limbs, Self-Organizing Feature Maps, and NoiseDriven Neuroplasticity. In (5), pp. 273-282.

45. Pearson, J.C., Finkel, L.H. and Edelman G.M. (1987) Plasticity in the organization of adult cerebral cortical maps: A computer simulation based on neuronal group selection. J. Neuroscience 7, 4209-4223.

46. Finkel, L.H., Pearson, J.C. and Edelman, G.M. (1997) Models of Topographic Map Organization. In: Pattern Formation in the Physical and Biological Sci- 
ences, H.F. Nijhout, L. Nadel, D. Stein, and F. Nijhout, eds. Addison-Wesley Reading, MA.

47. Mazza, M., de Pinho, M., Piqueira, J.R.C. and Roque, A.C. (2004) A Dynamical Model of Fast Cortical Reorganization. J. Comp. Neuroscience 16(2), 177-201.

48. Ramachandran, V.S. and Hirstein, W. (1998) The perception of phantom limbs. Brain 121, 1603-1630.

49. Aglioti, S., Cortese, F., Franchini, C. (1994) Rapid sensory remapping in the adult brain as inferred from phantom breast perception. Neuroreport 1994, 473-476.

50. Sptizer, M., Bohler, P., Weisbrod, M., Kishka, U. (1996). A neural network model of phantom limbs. Biological Cybernetics 72, 197-206.

51. McGonigle, D.J., Hänninen, R., Salenius, S., Hari, R., Frackowiak R.S.J. and Frith, C.D. (2002) Whose arm is it anyway? An fMRI case study of supernumerary phantom limb. Brain 125, 1265-1274.

52. Cohen, J.D., Romero, R.D., Servan-Schreiber, D. and Farah, M.J. (1994) Mechanisms of spatial attention: The relation of macrostructure to microstructure in parietal neglect. J. Cognitive Neuroscience 6, 377-387.

53. Thier, P. and Karnath, H.O., eds (1997) Parietal lobe contribution in orientation in $3 D$ space. Springer Verlag.

54. Rao, R.P.N. and Ballard, D.H. (1997) A computational model of spatial representations that explains object-centered neglect in parietal patients. In: Bower J.M., Ed., Computational Neuroscience: Trends in Research. Plenum Press, New York, NY.

55. Deco G, Rolls ET. (2002) Object-based visual neglect: a computational hypothesis. European J. Neuroscience 16(10), 1994-2000.

56. Deco, G. and Rolls, E.T. (2005) Neurodynamics of biased competition and cooperation for attention: a model with spiking neurons. J. Neurophysiology 94, 295313.

57. Morris, J.C., Storandt, M., Miller, J.P., McKeel, D.W., Price, J.L., Rubin E.H. and Berg, L. (2001) Mild cognitive impairment represents early-stage Alzheimer disease, Archives of Neurology 58, 397-405.

58. McClelland, J.L., McNaughton, B.L. and O'Reilly, R.C. (1995) Why there are complementary learning systems in the hippocampus and neocortex: insights from the successes and failures of connectionist models of learning and memory. Psychological Review 102, 419-457.

59. Hasselmo, M.E. and McClelland, J.L. (1999) Neural models of memory. Current Opinion in Neurobiology 9, 184-188.

60. Horn, D, Ruppin, E., Usher, M., Herrmann, M. (1993) Neural network modeling of memory deterioration in Alzheimer's disease. Neural Computation 5: 736-749. 
61. Ruppin, E, Reggia, J. (1995) A neural model of memory impairment in diffuse cerebral atrophy. British Journal of Psychiatry 166 (1), 19-28.

62. Horn, D., Levy, N., Ruppin, E. (1996) Neuronal-based synaptic compensation: A computational study in Alzheimer's disease. Neural Computation 8, 1227-1243.

63. Murre, J.M. (1996) TraceLink: A model of amnesia and consolidation of memory. Hippocampus 6, 675-684.

64. Meeter, M. and Murre, J.M.J. (2004) Simulating episodic memory deficits in semantic dementia with the TraceLink model. Memory 12, 272-287.

65. Meeter, M. and Murre, J.M.J. (2005) TraceLink: A model of consolidation and amnesia. Cognitive Neuropsychology 22(5), 559-587.

66. Hasselmo, M.E. (1991) Runaway synaptic modification in models of cortex: Implications for Alzheimer's disease. Neural Networks 7(1), 13-40.

67. Hasselmo, M.E. (1997) A computational model of the progression of Alzheimer's disease. MD Computing 14(3), 181-191.

68. Hasselmo, M.E. (1999) Neuromodulation: Acetylcholine and memory consolidation. Trends in Cognitive Sciences 3, 351-359.

69. Menschik, E.D. and Finkel, L.H. (1998) Neuromodulatory control of hippocampal function: Towards a model of Alzheimer's disease. Artificial Intelligence in Medicine 13, 99-121.

70. Menschik, E.D. and Finkel, L.H. (1999) Cholinergic neuromodulation and Alzheimer's disease: From single cells to network simulations. Progress in Brain Research 121, 19-45.

71. Menschik, E.D. and Finkel, L.H. (2000) Cholinergic neuromodulation of an anatomically reconstructed hippocampal CA3 pyramidal cell. Neurocomputing 3233, 197-205.

72. Menschik, E.D., Yen S.-C. and Finkel, L.H. (1999) Model and scale independent performance of a hippocampal CA3 network architecture. Neurocomputing 2627, 443-453.

73. Mesulam, M. (2004) The cholinergic lesion of Alzheimer's disease: pivotal factor or side show? Learning and Memory (Cold Spring Harbor, NY) 11, 43-49.

74. Duch, W. (2000) Therapeutic applications of computer models of brain activity for Alzheimer disease. J. Medical Informatics and Technologies 5, 27-34

75. Damasio A.R. (1996) Descartes' Error: Emotion, Reason and the Human Brain. Papermac.

76. Murre, J.M. (1997) Implicit and explicit memory in amnesia: some explanations and predictions by the TraceLink model. Memory 5(1-2), 213-232.

77. Romero, B. (1998) Self-Maintenance-Therapy (SMT) in early Alzheimer Disease. European Archives of Psychiatry and Clinical Neuroscience 248, 13-14. 
78. Pulvermuller, F. (2003) The Neuroscience of Language. On Brain Circuits of Words and Serial Order. Cambridge University Press, Cambridge, UK.

79. Andersen, P. and Moser, E.I. (1995) Brain temperature and hippocampal function. Hippocampus 5(6),491-8.

80. Haddadin, A.S., Abreu, M.M., Silverman, D.G., Luther, M. and Hines, R.L. (2005) Noninvasive Assessment of Intracranial Temperature Via the Medial Canthal-Brain Temperature Tunnel. Am. Soc. Anesthesiology Annual Meeting, Abstract A38.

81. Criswell, E. (1995) Biofeedback and Somatics. Freeperson Press, Novato, USA.

82. Newberg, A.B., Iversen, J. (2003) The neural basis of the complex mental task of meditation: neurotransmitter and neurochemical considerations. Med. Hypotheses 61(2), 282-91.

83. Banquet J.P, Gaussier P, Contreras-Vidal J.L, Gissler A, Burnod Y, Long D.L. (1998): A neural model of memory, amnesia and cortico-hippocampal interactions. In: Parks (6), pp. 77-120

84. Adeli, H., Ghosh-Dastidar, S. and Dadmehr, N. (2005) Alzheimer's disease and models of computation: Imaging, classification, and neural models. Journal of Alzheimer's Disease 7(3), 187-199.

85. Usui, S. (2003) Visiome: neuroinformatics research in vision project. Neural Networks 16, 1293-1300.

86. Wallenstein, G.V. and Hasselmo, M.E. (1997) Are there common neural mechanisms for learning, epilepsy, and Alzheimer's disease? In (22), pp. 314-346.

87. Weitzenfeld, A., Arbib, M.A. and Alexander, A. (2002) The Neural Simulation Language. A System for Brain Modeling. MIT Press, Cambridge, MA.

88. Fusi S., Del Giudice P. and Amit D.J. (2000) Neurophysiology of a VLSI spiking neural network. Proc. of the IEEE-INNS-ENNS International Joint Conference on Neural Networks, Vol. III, 121-126. 